\title{
Dor e fatores associados em pacientes atendidos em um serviço de urgência odontológica no sul do Brasil
}

\author{
Alessandra Noro Fernandes Barbosa*, Marcela Noro Fernandes Barbosa*, Cristiana Pereira Malta**, \\ Gisele Jung Franciscatto***, Jessye Melgarejo do Amaral Giordani****, Renata Dornelles \\ Morgental $* * * * *$ \\ * Estudante, Curso de Odontologia, Universidade Federal de \\ Santa Maria \\ ** Mestre em Ciências da Saúde e da Vida, Setor de \\ Atendimento Integral ao Estudante, Universidade Federal \\ de Santa Maria \\ *** Mestre em Endodontia, Curso de Odontologia, \\ Universidade Federal de Santa Maria \\ **** Doutor em Epidemiologia, Curso de Odontologia e \\ Programa de Pós-Graduação em Ciências Odontológicas, \\ Universidade Federal de Santa Maria \\ ***** Doutora em Endodontia, Curso de Odontologia e Programa \\ de Pós-Graduação em Ciências Odontológicas, \\ Universidade Federal de Santa Maria
}

Recebido: 21/02/2020. Aprovado: 25/06/2021.

\begin{abstract}
RESUMO
O objetivo deste estudo transversal foi avaliar o desfecho dor e fatores associados em pacientes atendidos em um serviço de urgência odontológica no sul do Brasil. Foram avaliados 137 prontuários provenientes de um projeto de extensão para capacitação em atendimento odontológico de urgência da Universidade Federal de Santa Maria (Santa Maria/RS), referentes ao período de abril de 2017 a dezembro de 2018. Os dados contidos na ficha clínica, autorrelatados pelos pacientes, foram coletados e variáveis relacionadas às características socioeconômicas, médicas e odontológicas foram submetidas à análise estatística descritiva e regressão de Poisson multivariada. A prevalência de dor nestes pacientes foi de $65,2 \%$ e a hipótese diagnóstica mais prevalente foi de pulpite aguda irreversível (46,2\% dos casos). A procura por atendimento foi maior na faixa etária entre 40-59 anos $(48,6 \%)$, no sexo feminino (64\%), em pacientes sem nível superior $(85,3 \%)$ e os dentes mais frequentemente tratados foram os posteriores $(82,7 \%)$. Houve associação entre a presença de dor e variáveis médicas, sendo que os pacientes com mais de duas doenças sistêmicas apresentaram maior prevalência de dor. $\mathrm{O}$ preenchimento inadequado dos prontuários odontológicos foi um achado comum, o que pode prejudicar o estabelecimento do perfil epidemiológico destes pacientes e o planejamento dos atendimentos futuros de forma eficiente, além de poder acarretar problemas jurídicos.

Descritores: Odontalgia. Epidemiologia. Pesquisa sobre Serviços de Saúde. Socorro de Urgência. Educação Odontológica.
\end{abstract}




\section{INTRODUÇÃO}

A condição precária de saúde bucal da população brasileira é um reflexo da situação socioeconômica, cultural e da desigualdade de acesso à assistência odontológica. Grande parte da população recorre a serviços odontológicos oferecidos de forma gratuita, por órgãos públicos e instituições de ensino, devido ao alto custo da prática privada ${ }^{1}$. Muitas vezes, os serviços de urgência odontológica funcionam como "porta de entrada" do paciente no sistema público de saúde, principalmente para aqueles que não conseguem acessar o serviço odontológico rotineiramente, para um acompanhamento longitudinal de sua saúde bucal ${ }^{2,3}$.

$\mathrm{Na}$ Odontologia, as urgências são extremamente comuns, principalmente aquelas ocasionadas por agentes agressores da polpa dental, causando uma dor difícil de $\operatorname{suportar}^{4}$ e um elevado grau de ansiedade ${ }^{5}$. Tal dor é consequência de reações inflamatórias que a polpa manifesta quando agredida, sendo a cárie dentária o principal fator etiológico ${ }^{6,7,8}$. Assim, a maioria das urgências odontológicas é desencadeada por alterações pulpares (pulpopatias) ou alterações do periodonto apical (periapicopatias) ${ }^{1,2,6,8,9}$.

Outras condições também podem levar os pacientes a procurar um serviço de urgência. De acordo com a Nota Técnica da Gerência de Vigilância e Monitoramento em Serviços de Saúde/ Gerência Geral de Tecnologia em Serviços de Saúde/ Agência Nacional de Vigilância Sanitária $N^{\circ}$ 04/2020, desenvolvida devido à pandemia pelo novo coronavírus (SARS-CoV-2), são classificados como procedimentos odontológicos de urgências: dor odontogênica aguda (pulpite), pericoronarite, alveolite, abscessos dentários ou periodontais, fratura dentária que resulta em dor ou trauma de tecidos moles bucais, necessidade de tratamento odontológico prévio a procedimento médico crítico, cimentação de coroas e próteses fixas, biópsias, ajustes de órteses e próteses que estejam causando dor e/ou comprometimento da função mastigatória, finalização de tratamento ou troca de medicação intracanal, remoção de lesões de cárie extensas ou restaurações que estejam causando dor, tratamento de necroses teciduais, mucosites e trauma dentário com avulsão ou luxação ${ }^{10}$.

A queixa principal na maioria dos atendimentos de urgência é a presença de dor ${ }^{1,6}$. A dor é conceituada como um fenômeno complexo e como uma experiência multidimensional, a qual envolve respostas sensoriais, além de aspectos cognitivos, emocionais, conceituais, culturais e motivacionais ${ }^{11}$. Diversos fatores locais $\mathrm{e}$ sistêmicos como idade, sexo, estado geral de saúde, grupo dentário afetado, estado pulpar e periapical, contatos oclusais, entre outros, podem interagir e modular a ocorrência de dor odontológica $^{12}$. Pacientes que se queixam de dor necessitam de atendimento imediato, uma vez que a dor atua negativamente sobre a qualidade de vida dos mesmos, ocasionando sofrimento, queda no desempenho profissional, no aprendizado, além de dificuldades no convívio social ${ }^{13}$.

Neste contexto, é de suma importância estudar as situações de urgências em Odontologia, pois elas são uma realidade vivida no cotidiano da clínica odontológica ${ }^{14}$. O cirurgião-dentista deve conhecer as características clínicas e radiográficas das patologias, para a determinação de um correto diagnóstico e, consequentemente, um adequado manejo dos pacientes que procuram $o$ atendimento de urgência ${ }^{6}$. Assim, é razoável supor que a educação odontológica dos cirurgiões-dentistas possibilita que os mesmos tenham contato com indivíduos portadores de quadros agudos de dor ${ }^{15}$. Porém, existe uma lacuna na formação dos profissionais de Odontologia quanto ao diagnóstico diferencial e abordagem em situações de urgência no consultório odontológico ${ }^{16}$.

Diversos estudos já foram realizados com o 
intuito de traçar o perfil epidemiológico de pacientes atendidos em serviços de urgência odontológica no Brasil ${ }^{1,2,8,9,17}$ e em outros países $^{3,18-20}$. Apesar de algumas semelhanças nos resultados, diferenças marcantes são identificadas e podem ser atribuídas à heterogeneidade das populações avaliadas, localidade da coleta (clínicas de ensino odontológico, hospitais universitários, pronto-atendimentos ou atenção primária), período e forma de coleta dos dados e características socioeconômicas da região ${ }^{21}$. A análise dos dados de tais pesquisas pode auxiliar no desenvolvimento de estratégias para melhoria dos serviços de urgência odontológica, em especial nos cursos de Odontologia, promovendo atendimentos de qualidade e com compromisso social ${ }^{22}$.

Apesar dos estudos supracitados, percebese que ainda existem poucos dados governamentais e informações na literatura referentes à caracterização da demanda e à associação da presença de dor no atendimento de urgência com variáveis relacionadas às características socioeconômicas, médicas e odontológicas. Tal fato justifica a realização da presente pesquisa, que avaliou o desfecho dor e fatores associados em pacientes atendidos em um serviço de urgência vinculado ao Curso de Odontologia da Universidade Federal de Santa Maria, em Santa Maria/RS, Brasil.

\section{METODOLOGIA}

O presente estudo foi previamente aprovado pelo Comitê de Ética em Pesquisa (CEP) da da Universidade Federal de Santa Maria (UFSM), CAEE: 02114818.4.0000.5346). A amostra deste estudo transversal foi composta por 137 prontuários de pacientes atendidos em um projeto de extensão para capacitação em atendimento odontológico de urgência da UFSM, localizada na cidade de Santa Maria, região central do estado do Rio Grande do Sul, no período de abril de 2017 a dezembro de 2018. Os critérios de elegibilidade na seleção da amostra foram: possuir a ficha clínica padrão do projeto de extensão preenchida e a devida assinatura do termo de consentimento para o uso de informações com fins acadêmicos.

Os dados contidos na ficha clínica, autorrelatados pelos pacientes, foram coletados e variáveis relacionadas às características socioeconômicas, médicas e odontológicas foram submetidas à análise estatística. Para análise dos dados, as variáveis foram categorizadas em faixa etária (0-19 anos; 20-39 anos; 40-59 anos; $\geq 60$ anos) $)^{23-25}$, sexo (feminino; masculino), escolaridade (nível superior; sem nível superior), doenças sistêmicas associadas (pacientes que não declararam possuir nenhum tipo de doença; com 1 doença; 2 ou mais doenças) e tipo de dente afetado (anterior; posterior). Em relação à história médica, foram observadas as seguintes condições patológicas: diabetes mellitus, hipertensão, cardiopatias, câncer, anemias, hemofilias, hepatites, Síndrome da Imunodeficiência Adquirida, problemas gástricos, problemas renais, osteoporose, tuberculose, doenças neurológicas ou psíquicas (depressão, epilepsia e outros problemas psiquiátricos).

Os dados foram analisados com a utilização do programa STATA 14 (Stata Corporation, College Station, TX, USA). A presença de dor ao chegar no atendimento de urgência foi o desfecho do estudo. Inicialmente, foi realizada uma análise descritiva das variáveis com os resultados expressos em frequências absolutas e relativas (tabela 1). Além da análise descritiva, análises não ajustadas foram realizadas para fornecer estatísticas resumidas e avaliações preliminares da associação da presença de dor no atendimento de urgência com variáveis relacionadas às características socioeconômicas, médicas e odontológicas. O modelo final foi ajustado pelas variáveis preditoras finais (faixa etária, sexo, escolaridade, doença e tipo de dente), usando 
análise de regressão de Poisson multivariada. Os resultados foram expressos por razões de prevalência (RP) e seus respectivos intervalos de confiança de 95\% (IC 95\%). A construção do modelo foi realizada utilizando critérios teóricoepidemiológicos.

\section{RESULTADOS}

Um total de 137 pacientes compuseram a amostra, com idades variando de 15 a 95 anos e média de 42,59 $( \pm 16,52)$ anos. A prevalência de dor, relatada ao chegar no serviço urgência, foi de $65,2 \%$. As hipóteses diagnósticas predominantes foram pulpite aguda irreversível $(46,2 \%)$ e abscesso dentoalveolar agudo (13,2\%). Casos sem envolvimento endodôntico direto, ou seja, sem alterações pulpares ou periapicais compreenderam $17,9 \%$ da amostra e consistiam em fratura dentária, trauma dentário, cimentação de próteses, lesões de cárie, abscesso periodontal, pericoronarite e alveolite.

A tabela 1 traz a ocorrência de dor conforme as características socioeconômicas, médicas e odontológicas. É notável que há divergência entre o número de pacientes avaliados em cada variável e o número total da amostra. Isso deveu-se à falta de informações, ou seja, ao incompleto preenchimento dos respectivos campos em alguns prontuários. A partir das informações coletadas, constatou-se que a procura por atendimento foi maior na faixa etária entre $40-59$ anos $(48,6 \%)$, no sexo feminino $(64 \%)$ e em pacientes sem nível superior $(85,3 \%)$. Um total de $57,1 \%$ dos pacientes que relataram possuir duas ou mais doenças apresentaram dor no atendimento de urgência. Além disso, os dentes mais frequentemente tratados foram os posteriores $(82,7 \%)$.

Tabela 1. Ocorrência de dor conforme as características socioeconômicas, médicas e odontológicas

\begin{tabular}{lrr}
\hline & \multicolumn{2}{c}{ Dor } \\
Variáveis & Não & Sim \\
\cline { 2 - 3 } & \multicolumn{2}{c}{} \\
\hline Faixa etária $(n=136)$ & $11(69,2)$ & $5(30,8)$ \\
$0-19$ anos & $28(72,8)$ & $11(27,8)$ \\
$20-39$ anos & $38(58,2)$ & $28(41,8)$ \\
$40-59$ anos & $11(72,7)$ & $4(27,3)$ \\
$\geq 60$ anos & & \\
Sexo $(n=136)$ & $57(65,3)$ & $30(34,7)$ \\
Feminino & $32(65,1)$ & $17(34,9)$ \\
Masculino & $11(63,2)$ & $6(36,8)$ \\
Escolaridade $(n=116)$ & $71(71,4)$ & $18(28,6)$ \\
$\quad$ Nível superior & & $19(23,9)$ \\
Sem nível superior & $59(76,1)$ & $14(44,4)$ \\
Doenças sistêmicas $(n=135)$ & $17(55,6)$ & $15(57,1)$ \\
Nenhuma & $11(42,9)$ & \\
1 doença & & $10(43,7)$ \\
2 ou mais doenças & $12(56,3)$ & $35(33,7)$ \\
Tipo de dente $(n=127)$ & $70(66,3)$ & \\
Anterior & & \\
Posterior &
\end{tabular}


Conforme a tabela 2, na análise bruta, as variáveis pacientes que declararam possuir um tipo de doença sistêmica e pacientes com duas ou mais doenças foram associadas à maior presença de dor ao chegar no atendimento de urgência $(\mathrm{p}<0,05)$.
Após ajuste, pacientes com duas ou mais doenças apresentaram maior prevalência de dor $(\mathrm{p}<0,05)$, com prevalência 2,85 vezes maior de dor em comparação aos que não possuem nenhum tipo de doença.

Tabela 2. Análise bruta e ajustada da associação da presença de dor no atendimento de urgência com variáveis relacionadas às características socioeconômicas, médicas e odontológicas

\begin{tabular}{|c|c|c|c|c|}
\hline Variáveis & $\begin{array}{c}\text { RP (IC 95\%) } \\
\text { Bruta } \\
\end{array}$ & p & $\begin{array}{c}\text { RP (IC 95\%) } \\
\text { Ajustada }\end{array}$ & $\mathbf{p}$ \\
\hline \multicolumn{5}{|l|}{ Faixa etária } \\
\hline 0-19 anos & 1,0 & & 1,0 & \\
\hline 20-39 anos & $0,90(0,34-2,39)$ & 0,837 & $0,60(0,22-1,65)$ & 0,322 \\
\hline $40-59$ anos & $1,36(0,56-3,27)$ & 0,493 & $0,77(0,30-2,02)$ & 0,601 \\
\hline$\geq 60$ anos & $0,89(0,25-3,15)$ & 0,852 & $0,54(0,15-1,92)$ & 0,344 \\
\hline \multicolumn{5}{|l|}{ Sexo } \\
\hline Feminino & 1,0 & & 1,0 & \\
\hline Masculino & $1,00(0,60-1,69)$ & 0,986 & $1,33(0,77-2,29)$ & 0,306 \\
\hline \multicolumn{5}{|l|}{ Escolaridade } \\
\hline Nível superior & 1,0 & & 1,0 & \\
\hline Sem nível superior & $0,78(0,32-1,87)$ & 0,573 & $0,80(0,27-2,43)$ & 0,701 \\
\hline \multicolumn{5}{|l|}{ Doenças sistêmicas } \\
\hline Nenhuma & 1,0 & & 1,0 & \\
\hline 1 doença & $1,86(1,02-3,40)$ & $0,044^{*}$ & $1,70(0,82-3,50)$ & 0,152 \\
\hline 2 ou mais doenças & $2,39(1,35-4,22)$ & $0,003 *$ & $2,85(1,48-5,47)$ & $0,002 *$ \\
\hline \multicolumn{5}{|l|}{ Tipo de dente } \\
\hline Anterior & 1,0 & & 1,0 & \\
\hline Posterior & $0,77(0,41-1,44)$ & 0,415 & $0,85(0,45-1,58)$ & 0,606 \\
\hline
\end{tabular}

RP = Razão de Prevalência (modelo de Regressão de Poisson)

\section{DISCUSSÃO}

Estudos epidemiológicos em serviços de atendimento de urgência buscam ajudar nas tomadas de decisão para solucionar problemas específicos $^{26}$, além de auxiliar no planejamento de futuras intervenções em saúde individual e coletiva $^{27}$. Assim, o presente estudo avaliou o desfecho dor e fatores associados em pacientes atendidos em um serviço de urgência vinculado ao Curso de Odontologia da UFSM.

A partir das informações coletadas nos prontuários, constatou-se que a faixa etária da população que mais procurou atendimento de urgência foi aquela entre 40-59 anos $(48,6 \%)$ e a procura foi maior no sexo feminino (64\%). De um modo geral, indivíduos do sexo feminino procuram mais os serviços de saúde do que os do sexo oposto $^{28}$. Alguns estudos ${ }^{2,17,29,30}$ enfatizam que essa discrepância entre os sexos se deve ao maior interesse das mulheres em cuidar de seus dentes e de sua aparência. No Brasil, segundo dados do Instituto Brasileiro de Geografia e Estatística IBGE, a população feminina é maior do que a masculina, o que também poderia explicar os resultados apresentados pelo presente estudo ${ }^{31}$. No entanto, outros pesquisadores observaram que 
homens foram acometidos mais frequentemente por situações de urgência odontológica do que mulheres ${ }^{32,33}$. Essa variedade de resultados entre os estudos pode ser atribuída à heterogeneidade das populações estudadas e às características socioeconômicas dos locais onde os dados foram coletados $^{21}$.

A literatura tem relatado uma associação entre condição de saúde bucal e perfil socioeconômico, mais especificamente, a influência do nível educacional na atitude da população em buscar atendimento odontológico $^{22,34}$. Os níveis de escolaridade mais baixos relacionam-se às piores condições de saúde bucal, pois parecem reduzir a preocupação com os agravos das doenças e os cuidados preventivos ${ }^{35}$. A maior parte da população que procurou atendimento de urgência no presente estudo não apresenta ensino superior (85,3\%), o que demonstra um baixo perfil socioeconômico. Esse fato vai ao encontro de resultados obtidos em outras pesquisas, mostrando que o nível socioeconômico inferior ${ }^{36}$ e a dificuldade financeira ${ }^{37}$ são fatores que motivam a procura por serviços de urgência odontológica gratuitos.

Os dentes mais frequentemente tratados foram os posteriores $(82,7 \%)$, o que também foi observado por um estudo realizado no Centro de Especialidades Odontológicas de Endodontia da Universidade Federal do Rio Grande do $\mathrm{Sul}^{38}$. Em tal pesquisa, os dentes posteriores corresponderam a $60,3 \%$ da amostra. Tais achados podem ser explicados pela complexa anatomia dos dentes posteriores e pela dificuldade de acesso, o que prejudica a realização de uma correta higienização, provocando maior prevalência de cárie e, consequentemente, de patologias pulpares/ periapicais ${ }^{39}$.

Apesar da redução na prevalência de cárie dentária no Brasil, uma grande parcela da população, principalmente aqueles que não têm assistência odontológica garantida, procura tratamento odontológico de urgência para alívio da dor $^{7}$. Assim, a dor é o principal motivo da busca por atendimento de urgência odontológica. Conforme descrito em estudo prévio, a maioria dos casos de dor de origem odontológica é causada por problemas endodônticos, dentre os quais a pulpite irreversível sintomática é o mais comum e, consequentemente, o tratamento mais realizado é a pulpectomia, demonstrando ainda a presença significante da cárie dentária na população ${ }^{1}$. No presente estudo, houve necessidade de tratamento endodôntico em 70,5\% dos casos atendidos e o diagnóstico de pulpite irreversível sintomática foi observado em $46,2 \%$ dessas ocorrências.

Apesar do tratamento realizado durante o atendimento de urgência apresentar caráter temporário, o paciente precisa ter sua queixa principal resolvida e, em seguida, ser encaminhado para a continuidade do tratamento ${ }^{40}$. Assim, na prática clínica, é comum o cirurgião-dentista se deparar com pacientes portadores de quadros agudos de dor de diferentes origens. No entanto, devido ao processo de triagem realizado nos cursos de Odontologia, muitas vezes o aluno de graduação não tem contato com o indivíduo em crise de dor. O projeto de extensão avaliado no presente estudo permite a vivência dos graduandos com casos dessa natureza, contribuindo substancialmente para sua formação acadêmica ${ }^{15}$.

A clínica de urgência pode ser desenvolvida dentro dos cursos ou em serviços de saúde conveniados e é uma experiência enriquecedora para os graduandos de Odontologia, pois permite que desenvolvam a capacidade diagnóstica, uma vez que estarão atendendo pacientes não triados, ou seja, não saberão qual a necessidade de tratamento do paciente, a priori. De forma geral, os estudantes apresentam dificuldade em estabelecer diagnóstico, pelo fato da fragmentação das disciplinas: diagnóstico em periodontia, em endodontia, em odontopediatria, em cirurgia, entre outras $^{15,41}$. 
Deve-se ressaltar que as clínicas de urgência nos cursos também apresentam limitações. Por ser, na maioria das vezes, o primeiro contato do estudante com o paciente em crise de dor e existir a necessidade de um rápido diagnóstico, raciocínio clínico e tomada de decisão, tais atitudes acabam recaindo sobre o professor. Assim, as clínicas de urgência no ambiente de ensino acabam não sendo o ideal para permitir uma adequada educação odontológica, com o desenvolvimento no graduando dessas competências e habilidades. $\mathrm{O}$ impacto sobre a formação dos cirurgiões-dentistas é maior quando o estágio acontece em serviços de saúde, os quais permitem evidenciar a relação da ocorrência das urgências com as situações de vida dos pacientes ${ }^{41}$.

Diversos fatores sistêmicos, entre eles o estado geral de saúde, podem modular a ocorrência de dor odontológica ${ }^{12}$. No presente estudo, pacientes com 2 ou mais doenças apresentaram maior prevalência de dor. Esse resultado pode ser explicado pelo conhecimento estabelecido de que pacientes com maior carga de doenças crônicas têm predisposição a infeções odontogênicas ${ }^{42}$. Evidências sugerem que os níveis séricos de marcadores inflamatórios estão aumentados em pacientes com doenças cardiovasculares ${ }^{43}$, bem como em pacientes com periodontite apical ${ }^{44}$. Além disso, uma relação entre marcadores inflamatórios e dor também é sugerida ${ }^{45}$. Deve-se considerar que o processo saúde-doença é multicausal, ou seja, múltiplos fatores interrelacionados em redes de causalidade estão atribuídos à gênese das doenças ${ }^{5}$.

Nas últimas décadas, uma questão recorrente às pesquisas científicas diz respeito à interação saúde bucal-doença sistêmica, em que o comprometimento imunológico causado pela alteração sistêmica pode contribuir para a manutenção de doenças bucais ${ }^{46,47,48}$. Outra possível explicação para a maior prevalência de dor em pacientes com comprometimento sistêmico relaciona-se ao perfil psicológico de extrema ansiedade de tais pacientes. Eventos psicológicos, emocionais e comportamentais têm também a capacidade de transformar a compreensão e interpretação dos estímulos dolorosos, sendo pacientes fóbicos mais susceptíveis à dor ${ }^{49}$. As razões biológicas para o achado do presente estudo não estão totalmente esclarecidas e precisam ser avaliadas em futuras pesquisas projetadas especificamente para testar a hipótese da influência do estado geral de saúde na dor de origem dentária.

Por fim, é necessário destacar a divergência entre o número de pacientes avaliados em cada variável e o número total da amostra $(n=137)$. Isso deu-se pela ausência de campos preenchidos ou preenchidos de forma errônea. Essa falha no preenchimento dos prontuários odontológicos também foi relatada em outros estudos ${ }^{6,38}$. O correto preenchimento dos prontuários é fundamental para que se conheça as demandas da instituição de ensino, traçando o perfil dos pacientes e potencializando a resolutividade do serviço. $O$ acadêmico também deve estar consciente dos aspectos legais relacionados aos prontuários e da necessidade de um preenchimento padronizado, abrangendo desde a anamnese até o plano de tratamento e descrição dos procedimentos, evitando assim problemas jurídicos futuros ${ }^{38,50}$.

Estudos que traçam o perfil da população atendida nas clínicas dos cursos de Odontologia, associando-o com desfechos clínicos, permitem prever as principais demandas nos atendimentos de urgência. Essas informações são de grande valia para avaliar a importância do serviço de urgência na instituição envolvida, para melhorar a logística de atendimento e analisar o processo educativo do curso. O reflexo disso é a formação de cirurgiõesdentistas capacitados para o manejo de situações de urgência odontológica e, segundo as Diretrizes Curriculares Nacionais do Curso de Graduação em Odontologia $^{51}$, pautados em princípios éticos, 
legais e na compreensão da realidade social, cultural e econômica do seu meio, dirigindo sua atuação para a transformação da realidade em benefício da sociedade

\section{CONCLUSÃO}

O perfil epidemiológico dos pacientes atendidos no serviço de urgência vinculado ao Curso de Odontologia da UFSM inclui pacientes com idade entre 40-59 anos, do sexo feminino, sem nível superior, com comprometimento de dentes posteriores na maior parte da amostra. Houve associação entre dor e variáveis médicas, sendo que os pacientes com mais de duas doenças sistêmicas apresentaram maior prevalência de dor ao chegar no atendimento de urgência. A importância do completo preenchimento dos prontuários odontológicos deve ser constantemente reforçada nos cursos de Odontologia, sendo fundamental para traçar corretamente o perfil epidemiológico dos pacientes e programar os atendimentos futuros de forma eficiente, além de prevenir possíveis problemas jurídicos.

\section{ABSTRACT \\ Pain and associated factors in patients attending an emergency dental service in southern Brazil}

This cross-sectional study aimed to evaluate the pain outcome and associated factors in patients attending an emergency dental service in southern Brazil. One hundred and thirty-seven (137) patient records from an extension project for emergency dental care training at the Universidade Federal de Santa Maria (Santa Maria, RS, Brazil), relative to the period from April 2017 to December 2018, were evaluated. The data in the records, self-reported by the patients, were collected, and variables related to socioeconomic, medical and dental characteristics were subjected to descriptive statistical analysis and multivariate Poisson regression. The prevalence of pain in these patients was $65.2 \%$, and the most prevalent diagnostic hypothesis was irreversible acute pulpitis (46.2\% of the cases). The demand for care was greater in the age group between 40-59 years $(48.6 \%)$, in women $(64 \%)$, in patients without university education $(85.3 \%)$, and posterior teeth were the most frequently treated $(82.7 \%)$. There was an association between the presence of pain and medical variables, being that patients with more than two systemic diseases had a higher prevalence of pain. The inadequate completion of dental records was a common finding, which can impair the establishment of the epidemiological profile of these patients and the efficient planning of future dental care services, as well as cause legal problems.

Descriptors: Toothache. Epidemiology. Health Services Research. Emergency Relief. Endodontics.

\section{REFERÊNCIAS}

1. Martins EP, Oliveira OR, Bezerra SRS, Dourado AT. Estudo epidemiológico de urgências odontológicas da FOP/UPE. RFO UPF. 2014;19(3):316-22.

2. Cassal JB, Cardoso DD, Bavaresco CS. Perfil dos usuários de urgência odontológica em uma unidade de atenção primária à saúde. Rev APS. 2011;14(1):85-92.

3. Lewis C, Lynch H, Johnston B. Dental complaints in emergency departments: a national perspective. Ann Emerg Med. 2003;42(1):93-9.

4. Menini MO, Gomes BPFA, Ferraz CCR, Souza-Filho FJ, Zaia AA. Avaliação do sucesso clínico do atendimento de urgência endodôntica na FOP - Unicamp. Rev Assoc Paul Cir Dent. 2007;61(1):39-43.

5. Kanegane K, Penha SS, Borsatti MA, Rocha RG. Dental anxiety in an emergency dental service. Rev Saúde Públ. 2003;37(6):78692.

6. Munerato MC, Fiaminghi DL, Petry PC. Urgência em Odontologia: um estudo 
retrospectivo. Rev Fac Odontol Porto Alegre. 2005;46(1):90-5.

7. Shqair AQ, Gomes GB, Oliveira A, Goettems ML, Romano AG, Schardozim LR, et al. Dental emergencies in a university pediatric dentistry clinic: a retrospective study. Braz Oral Res. 2012;26(1):50-6.

8. Tortamano IP, Leopoldino VD, Borsatti MA, Penha SS, Buscariolo IA, Costa CG, et al. Aspectos epidemiológicos e sociodemográficos do setor de urgência da Faculdade de Odontologia da Universidade de São Paulo. RPG Rev Pós-Grad. 2006;13(4):299-306.

9. Dourado AT, Martins EP, De Oliveira OR, Bezerra SRS. Estudo epidemiológico de urgências odontológicas. JBC J Bras Clin Odontol Integr. 2005;9(48):60-4.

10. Agência Nacional de Vigilância Sanitária ANVISA. Nota Técnica GVIMS/GGTES/ ANVISA $N^{\circ}$ 04/2020. Orientações para serviços de saúde: medidas de prevenção e controle que devem ser adotadas durante a assistência aos casos suspeitos ou confirmados de infecção pelo novo coronavírus (SARS-CoV-2). 2020. [Acesso em 23 mar. 2021]. Disponível em: https://www.gov.br/anvisa/pt-br/centrais deconteudo/publicacoes/servicosdesaude/no tas-tecnicas/nota-tecnica-n-04-2020-gvimsggtes-anvisa-atualizada.pdf/view.

11. Sessle BJ. Recent developments in pain research: central mechanisms of orofacial pain and its control. J Endod. 1986;12(10):435-44.

12. Hargreaves KM, Cohen S, Berman LH. Cohen's Pathways of the Pulp. 10th ed. St. Louis, Mo.: Mosby Elsevier; 2011.

13. Alexandre GC, Nadanovsky P, Lopes CS, Faerstein E. Prevalência e fatores associados à ocorrência da dor de dente que impediu a realização de tarefas habituais em uma população de funcionários públicos no Rio de Janeiro, Brasil. Cad Saúde Pública. 2006;22(5):1073-78.

14. Sanchez HF, Drumond MM. Atendimento de urgências em uma Faculdade de Odontologia de Minas Gerais: perfil do paciente e resolutividade. RGO. 2011; 59(1):79-86.

15. Marchini L, Patrocínio MC, Rode SM. Plano de ensino de uma disciplina de "urgências e emergências em Odontologia”. Pós-Grad Rev Fac Odontol São José dos Campos. 2000;3(1):105-12.

16. Hanna LMO, Alcântara HSC, Damasceno JM, Santos MTBR. Conhecimento dos Cirurgiões Dentistas diante Urgência/ Emergência Médica. Rev Cir Traumatol Buco-Maxilo-Fac. 2014;14(2):79-80

17. De Paula JS, De Oliveira M, Soares MRSP, Chaves MAMC, Mialhe FL. Perfil epidemiológico dos pacientes atendidos no Pronto Atendimento da Faculdade de Odontologia da Universidade Federal de Juiz de Fora. Arq Odontol. 2012;48(4):257262.

18. Hocker MB, Villani JJ, Borawski JB, Evans CS, Nelson SM, Gerardo CJ, et al. Dental visits to a North Carolina Emergency Department: a painful problem. N C Med J. 2012;73(5):346-51.

19. Quinonez C, Gibson D, Jokovic A, Locker D. Emergency department visits for dental care of nontraumatic origin. Community Dent Oral Epidemiol. 2009;37(4):366-71.

20. Von Kaenel D, Vitangeli D, Casamassimo PS, Wilson S, Preisch J. Social factors associated with pediatric emergency department visits for caries-related dental pain. Pediatr Dent. 2001;23(1):56-60.

21. Fonseca DAV, Mialhe FL, Ambrosano GMB, Pereira AC, Meneghim MC. Influência da organização da atenção básica 
e das características sociodemográficas da população na demanda pelo pronto atendimento odontológico municipal. Ciênc Saúde Colet. 2014;19(1):269-77.

22. Brandini DA, Poi WR, Mello MLM, Macedo APA, Panzarini SR, Pedrini D et al. Caracterização social dos pacientes atendidos na disciplina de Clínica Integrada da Faculdade de Odontologia de Araçatuba, UNESP. Pesqui Bras Odontopediatria Clín Integr. 2008;8(2):245-50.

23. Reis DC, Almeida TAC, Quites HFO, Sampaio MM. Perfil epidemiológico da tuberculose no Município de Belo Horizonte (MG), no período de 2002 a 2008. Rev Bras Epidemiol. 2013;16(3):592-602

24. Santos AD, Silva CRA, Medeiros JD, Panazzolo GLG, Silva HCTA, Filho AAMR, et al. Perfil epidemiológico de pacientes com diabetes mellitus. Braz J Surg Clin Res. 2018;4(2):40-6.

25. Alves RH, Reis DC, Viegas AM, Neves JAC, Almeida TAC, Flisch TMP. Perfil epidemiológico da AIDS em Contagem, Minas Gerais, Brasil entre 2007 e 2011. Rev Epidemiol Control Infect. 2015;5(3):147-52.

26. Tigre CH, Plaut R, Libel M, Castellanos PL. La práctica epidemiológica en los sistemas de servicios de salud. Educ Méd Salud. 1990;24(3):306-20.

27. Paim JS. Epidemiologia e planejamento: a recomposição das práticas epidemiológicas na gestão do SUS. Ciênc Saúde Colet. 2003;8(2):557-67.

28. Pinheiro RS, Viacava F, Travassos C, Brito AS. Gênero, morbidade, acesso e utilização de serviços de saúde no Brasil. Ciênc Saúde Colet. 2002;7(4):687-707.

29. Pinto EC, Barros VJ, Coelho MQ, Costa SMC. Urgências odontológicas em uma Unidade de Saúde vinculada à Estratégia Saúde da Família de Montes Claros, Minas
Gerais. Arq Odontol. 2012;48(3):166-74.

30. Flumignan JDP, Neto LFS. Atendimento odontológico em unidades de emergência: caracterização da demanda. Rev Bras Odontol. 2014;71(2):124-29.

31. Matos C. Brasil tem 96 homens para cada 100 mulheres aponta censo [Internet]. 2012 [Citado 2016 outubro 11]. Disponível em: http://www1.folha.uol.com.br/cotidiano/90 8784-brasil-tem-96-homens-para- cada100-mulheres-aponta-censo

32. Segura JJ, Jiménez RA. Urgências endodônticas: análise epidemiológica de 80 casos. J Endod Pract. 2002;1(2):16-23.

33. Tramini P, Nassar BAQ, Valcarcel J, Gilbert P. Factors associated with the use of emergency dental care facilities in a French public hospital. Spec Care Dentist. 2010;30(2):66-71.

34. Padilha WWN, Carvalho DM, Oliveira V, Amaral MF, Martinho DS. Associação entre indicadores de saúde bucal e nível soocioeconômico em pacientes da Clínica Integrada. Pesq Bras Odontopediatria Clín Integr. 2001;1(1):31-44.

35. Baldani MH, Vasconcelos AGG, Antunes JLF. Associação do índice CPO-D com indicadores socioeconômicos e de provisão de serviços odontológicos no Estado do Paraná, Brasil. Cad Saúde Pública. 2004;20(1):143-152.

36. Lacerda JT, Simionato EM, Peres KG, Peres MA, Traebert J, Marcenes W. Dor de origem dental como motivo de consulta odontológica em uma população adulta. Rev Saúde Públ. 2004;38(3):453-58.

37. Mialhe FL, Possobon RF, Boligon F, Menezes MA. Medo odontológico entre pacientes atendidos em um serviço de urgência. Pesq Bras Odontopediatria Clín Integr. 2010;10(3):483-87.

38. Pandolfo MT, Giordani JMA, Neves M, 
Soares RG. CEO-Endodontia da UFRGS: um estudo transversal sobre a prevalência de atendimentos, características dos pacientes e documentação dos prontuários. Rev ABENO. 2015;15(4):67-77.

39. Fernandes LMPSR, Zapata RO, Duarte MAH, Capelozza ALA. Prevalence of apical periodontitis detected in cone beam CT images of a Brazilian subpopulation. Dentomaxillofac Radiol. 2013;42(1):2-7.

40. Albuquerque YE, Zuanon ACC, Pansani CA, Giro EMA, Lima FCBA, Pinto LAMS, et al. Perfil do atendimento odontológico no Serviço de Urgência para crianças e adolescentes da Faculdade de Odontologia de Araraquara (FOAr) - UNESP. Rev Odontol UNESP. 2016;45(2):115-20.

41. Werneck MAF, Senna MIB, Drumond MM, Lucas SD. Nem tudo é estágio: contribuições para debate. Ciênc Saúde Colet. 2010;5(1):221-31.

42. Cachovan G, Phark JH, Schon G, Pohlenz P, Platzer U. Odontogenic infections: an 8-year epidemiologic analysis in a dental emergency outpatient care unit. Acta Odontol Scand. 2013;71(3):518-24.

43. Blake GJ, Ridker PM. Inflammatory biomarkers and cardiovascular risk prediction. $\mathrm{J}$ Intern Med. 2002;252(4):283-94.

44. Gomes MS, Blattner TC, Sant'Ana Filho M, Grecca FS, Hugo FN, Fouad AF, et al. Can apical periodontitis modify systemic levels of inflammatory markers? A systematic review and meta-analysis. $\mathrm{J}$ Endod. 2013;39(10):1205-17.

45. Oliveira KG, von Zeidler SV, Lamas AZ, Podestá JRV, Sena A, Souza ED, et al. Relationship of inflammatory markers and pain in patients with head and neck cancer prior to anticancer therapy. Braz J Med Biol Res. 2014;47(7), 600-4.

46. Berlin-Broner Y, Febbraio M, Levin L.
Association between apical periodontitis and cardiovascular diseases: a systematic review of the literature. Int Endod J. 2017;50(9):847-59.

47. Khalighinejad N, Aminoshariae MR, Aminoshariae A, Kulild JC, Mickel A, Fouad AF. Association between Systemic Diseases and Apical Periodontitis. J Endod. 2016;42(10):1427-3.

48. Segura-Egea JJ, Martín-González J, Castellanos-Cosano L. Endodontic medicine: connections between apical periodontitis and systemic diseases. Int Endod J. 2015;48(10):33-51.

49. Madan GA, Madan SG, Madan AD. Failure of alveolar nerve block: exploring alternatives. J Am Dent Assoc. 2002;133(7):843-46.

50. Benedicto EM, Lages LHR, Oliveira OF, Silva RHA, Paranhos LR. A importância da correta elaboração do prontuário odontológico. Odonto (São Bernardo do Campo). 2010;18(36):41-50.

51. Brasil. Ministério da Educação. Conselho Nacional de Educação. Câmara de Educação Superior. Resolução n ${ }^{\circ} .3$, de 19 de fevereiro de 2002. Diretrizes Curriculares Nacionais do Curso de Graduação em Odontologia. Diário Oficial da União, Poder Executivo, Brasília, DF, 04 de março de 2002. [Acesso em 23 mar. 2021]. Disponível em: http://portal.mec.gov.br/cne/arquivos/pdf/C ES032002.pdf.

\section{Correspondência para:}

Cristiana Pereira Malta

e-mail: cris_malta@hotmail.com

Curso de Odontologia

Universidade Federal de Santa Maria

Av. Roraima, $n^{\circ}$ 1000, Prédio 26F, Sala 2284

Cidade Universitária, Camobi

97105-900, Santa Maria/RS 